\title{
The 12th Asilomar Conference on Mass Spectrometry, September 20 - 24, 1996 "Elemental Mass Spectrometry," Gary M. Hieftje, Program Chair The Asilomar Conference Center, Pacific Grove, California
}

\begin{tabular}{|c|c|}
\hline Speaker & Conference Presentations \\
\hline $\begin{array}{l}\text { Ramon Barnes } \\
\text { Univ. of Massachusetts }\end{array}$ & Capillary Electrophoresis with ICP Mass Spectrometry \\
\hline $\begin{array}{l}\text { Bruce Bushaw } \\
\text { Pacific NW Nai l Lab. }\end{array}$ & Resonance lonization-Mass Spectrometry \\
\hline $\begin{array}{l}\text { Joseph Caruso } \\
\text { Univ. of Cincinnati }\end{array}$ & New Approaches to Elemental Speciation with Plasma Mass Spectrometric Detection \\
\hline $\begin{array}{l}\text { Don Douglas } \\
\text { Univ. of British Columbia }\end{array}$ & ICP - Mass Spectrometry with Quadrupoles Operated in Alternate Stability Regions \\
\hline $\begin{array}{l}\text { Douglas Duckworth } \\
\text { Oak Ridge Nat'l Lab. }\end{array}$ & Developments in Glow Discharge Ion Trap Mass Spectrometry \\
\hline $\begin{array}{l}\text { Chris Enke } \\
\text { Univ. of New Mexico }\end{array}$ & Fundamental Studies in ESI-Mass Spectrometry \\
\hline $\begin{array}{l}\text { John Eyler } \\
\text { Univ. of Florida }\end{array}$ & Recent Developments in Elemental Analysis with FTICR Detection \\
\hline Conrad Gregoire & Electrothermal Vaporization Sample Introduction for ICP Mass Spectrometry: \\
\hline $\begin{array}{l}\text { Geological Survey Canada } \\
\text { Alex Halliday } \\
\text { Univ. of Michigan }\end{array}$ & $\begin{array}{l}\text { Current Status and Future Prospects } \\
\text { Isotope-Ratio Mass Spectrometry }\end{array}$ \\
\hline $\begin{array}{l}\text { Willard Harrison } \\
\text { Univ. of Florida }\end{array}$ & The Microsecond Glow Discharge as an Ion Source for Atomic Mass Spectrometry \\
\hline $\begin{array}{l}\text { Gary M. Hieftje } \\
\text { Indiana Univ. }\end{array}$ & New Directions in Atomic Mass Spectrometry \\
\hline $\begin{array}{l}\text { Gary Horlick } \\
\text { Univ. of Alberta }\end{array}$ & Inorganic Speciation by Electrospray Mass Spectrometry: Fact or Fiction? \\
\hline $\begin{array}{l}\text { R. Sam Houk } \\
\text { lowa State Univ. }\end{array}$ & $\begin{array}{l}\text { Improving Precision and Accuracy for the Direct Analysis of Solids by Laser Ablation } \\
\text { Inductively Coupled Plasma-Mass Spectrometry }\end{array}$ \\
\hline $\begin{array}{l}\text { David Koppenaal } \\
\text { Pacific NW Nat'l Lab. }\end{array}$ & New Directions in Atomic Mass Spectrometry: The Ion Trap Way \\
\hline $\begin{array}{l}\text { Ted Litherland } \\
\text { Univ. of Toronto }\end{array}$ & Accelerator Mass Spectrometry \\
\hline $\begin{array}{l}\text { Ken Marcus } \\
\text { Clemson Univ. }\end{array}$ & GDMS - Beyond Direct Solids Elemental Analysis \\
\hline $\begin{array}{l}\text { Akbar Montaser } \\
\text { George Washington Univ. }\end{array}$ & Helium ICP Mass Spectrometry \\
\hline $\begin{array}{l}\text { David Myers } \\
\text { Leco Corporation }\end{array}$ & On-Axis ICP Time of Flight Mass Spectrometry \\
\hline John Olesik & Time-Resolved Inductively Coupled Plasma Mass Spectrometry Measurements and the \\
\hline $\begin{array}{l}\text { Ohio State Univ. } \\
\text { Kimberly Prather }\end{array}$ & $\begin{array}{l}\text { Fate of Individual Sample Droplets } \\
\text { Aerosol Time of Flight Mass Spectrometry }\end{array}$ \\
\hline Univ. of California, Riverside & \\
\hline $\begin{array}{l}\text { Richard Russo } \\
\text { Lawrence Berkeley Lab. }\end{array}$ & Laser Ablation ICP Mass Spectrometry \\
\hline Robert Shaw & Isotopic Analysis of Inorganic Compounds by High Resolution Optical Spectroscopy \\
\hline Oak Ridge Nat'l Lab. & Using Diode Lasers \\
\hline $\begin{array}{l}\text { Scott Tanner } \\
\text { PE Sciex }\end{array}$ & Interface Design in ICP Mass Spectrometry \\
\hline
\end{tabular}

For additional information, contact

ASMS, 1201 Don Diego Avenue, Santa Fe, NM 87505. Phone: (505) 9894517.

The deadline for registration is August 16, 1996. 